\title{
Effects of P16 DNA Methylation on Proliferation, Senescence, and Lifespan of Human Fibroblasts
}

Running title: $P 16$ methylation prevents cell senescence

Ying Gan, Chenghua Cui, Shengyan Xiang, Baozhen Zhang*, Dajun Deng*

Key Laboratory of Carcinogenesis and Translational Research (MOE/Beijing), Division of Etiology,

Peking University Cancer Hospital and Institute, Fu-Cheng-Lu \#52, Haidian-District, Beijing, 100142,

China

${ }^{*}$ Corresponding authors

Email address:

Ying Gan, ganying1989@126.com

Chenghua Cui, rockcui@126.com

Shengyan Xiang, syxiang@gmail.com

Baozhen Zhang, zbz94@126.com

Dajun Deng, dengdajun@bjmu.edu.cn 


\section{ABSTRACT}

The aim is to study the effects of P16 DNA methylation on lifespan of normal cells. An expression-controllable PTRIPZ vector expressing $P 16$-specific zinc finger binding protein-based methyltransferase (P16-Dnmt) was used to induce $P 16$ methylation in primary CCD-18Co fibroblasts via stable transfection. Long-term dynamic IncuCyte analysis showed that CCD-18Co fibroblasts expressing baseline P16-Dnmt continued proliferating until passage- 26 in the $53^{\text {th }}$ post-transfection week, while vector control cells stopped proliferating at passage- 6 and completely died 2 weeks later. The proliferation rate of baseline P16-Dnmt cells was significantly higher than that of vector control cells. The proportion of $\beta$-galactosidase-positive staining cells was significantly decreased in baseline P16-Dnmt cells compared to vector control cells. The P16 expression was lost in baseline P16-Dnmt cells at and after passage-6. The average telomere length in baseline P16-Dnmt cells also gradually decreased. In conclusion, P16 methylation could prevent senescence, promote proliferation, and expand lifespan of human fibroblasts, which may play a role in cancer development.

Key words: DNA methylation/epigenetic editing/fibroblasts/P16 gene/cell senescence/transformation

Summary: A zinc finger protein-based DNA methyltransferase (P16-Dnmt) expressed the baseline level could specifically methylate $P 16$ promoter $C p G$ islands. $P 16$ methylation induced by baseline P16-Dnmt could significantly prevent senescence, promote proliferation, and expand lifespan of primary human fibroblasts. 


\section{INTRODUCTION}

P16 gene $(C D K N 2 A)$ is most frequently inactivated in cancer genomes by allele deletion and methylation of CPG islands around transcription start site (TSS) (1-5). P16 germline inactivation causes high susceptibility to familial melanoma and pancreatic cancer (6,7). Abnormal $P 16$ methylation is prevalent in cancer and precancerous tissues (5). P16 methylation inactivates transcription of genes, including $A N R / L$ located within the $C D K N 2 A / B$ gene antisense-strand $(8,9)$. Many studies showed that $P 16$ methylation significantly increased risk of malignant transformation of precancerous tissues such as epithelial dysplasia and Barrett's esophagus (10-15). These phenomenons imply that $P 16$ methylation maybe a driver in cancer development. However, solid evidence to prove that epigenetic $P 16$ inactivation drives malignant transformation of normal cells is still needed.

Recently, epigenetic editing tools with different editing efficiencies and specificities are emerging which are based on engineered ZFP (zinc finger protein), CRISPR (clustered regularly interspaced short palindromic repeats)/dCas9, and TALE (transcription activator-like effectors) (16-20). For example, ZFP-Dnmts could specifically methylate whole P16 CpG islands $(8,16,17)$; TALE-Dnmts could methylate both target P16 CpG islands and CpG islands within near regions (18); CRISPR/dCas9-Dnmts could focally methylate sgRNA target-flanking regions (50-bp, not including sgRNA target sequence) $(21,22)$. Although the construction efficiency for ZFP-based editing tools is lower than those for dCas9- and TALE-based tools, it is likely that ZFP-Dnmts is optimal to induce full methylation of whole target CpG islands.

It has been reported that extensive methylation of whole $P 16 \mathrm{CpG}$ islands could be stably 
maintained in cancer cells, whereas focal methylation of $P 16$ exon-1 is not stable (23). In the present study, extensive methylation of whole $P 16 \mathrm{CpG}$ islands was stably and specifically induced in normal human fibroblasts by baseline P16-specific ZFP-Dnmt (P16-Dnmt). We found that P16 methylation significantly prevented senescence, promoted proliferation, and prolonged lifespan of human fibroblasts.

\section{METHODS}

\section{Cell line and Culture}

The primary/early-passage normal human colon fibroblast CCD-18Co cell line was directly purchased from American Type Culture Collection (ATCC, CRL-1459) in March 2012. This cell line that was derived from normal human colon tissue from a 2.5-month old black female begins to senesce at subculture times 18 in our laboratory (the total population doubling time is about 42 according to ATCC specification description). Cells were cultured in ATCC-formulated EMEM medium (ATCC Cat. No. 30-2003) supplemented with $15 \%$ FBS (Gibco, USA), and maintained at $37^{\circ} \mathrm{C}$ in humidified air with $5 \% \mathrm{CO}_{2}$. Cells were subcultured with medium renewal every 2-3 days. When cell confluence reached $70-80 \%$, cells were subcultured at ratio of $1: 2-3$. Cells subcultured at the $8^{\text {th }}$ time in our laboratory (pre-experiment passage-8) were used in transfection experiments.

CCD-18Co cell line was tested and anthenticated by Genewiz, Inc Beijing on Sept. 9, 2014 when it was used in this study. Short tanden repeat (STR) patterns were analyzed using GenePrint 10 System (Promega). The data were analyzed using GeneMapper4.0 software and then compared with the ATCC databases for reference matching. This cell line is considered to be "identical" to the reference cell line CCD-18Co in the ATCC STR database, as the STR profile yields a $100 \%$ match. 


\section{P16-Dnmt and Control Vectors and Transfection}

The established P16-Dnmt and vector control pTRIPZ were prepared and used to transfect cells as previously described (8). Briefly, an engineered $P 16$ promoter-specific seven zinc finger protein (7ZFP) was fused with the catalytic domain of mouse dnmt3a, and integrated into the pTRIPZ vector containing a "Tet-On" switch. The purified P16-Dnmt plasmid DNA was mixed with pCMV-VSV-G and pCMV- $\Delta 8.9$ (Addgene, USA) to prepare lentivirus infection particles. When the confluence of CCD- 18 Co cells at pre-experiment passage- 8 reached approximately $40 \%$, these cells were infected with the P16-Dnmt or vector control virus particles, and incubated for 48 hours. Puromycin was added into the medium (final concentration, $0.5 \mu \mathrm{g} / \mathrm{mL}$ ) to remove non-transfected cells, and to maintain the transfected cells. The pooled cells treated with puromycin for two weeks were considered to be stably transfected cells.

\section{Cell Proliferation Analysis using IncuCyte Zoom}

Cell proliferation status was dynamically recorded using the IncuCyte $\mathrm{ZOOM}^{\mathrm{TM}}$ live-cell imaging platform. Each trial consisted of 6-9 parallel microwells, and all assays were repeated 2-3 times.

\section{Tumor Formation}

Baseline P16-Dnmt or vector control CCD-18Co cells $\left(5 \times 10^{5}\right.$ cells $\left./ 150 \mu \mathrm{L}\right)$ embedded with matrigel (BD Biosciences, NJ, USA) were subcutaneously injected (per site, 5 mice/group) into NOD-SCID mice (SPF-grade, female, 6-8 w, 20-22 g; purchased from Beijing HFK Biosci Co.LTD). Seventeen weeks after implantation, these mice were sacrificed, and the possible xenograft tumors were determined. The animal ethical committee at Peking University Cancer Hospital and Institute approved the experiment. 


\section{Bisulfite-DHPLC and -Sequencing}

Unmethylated cytosine residues in genomic DNA samples were modified with ZMYO EZ Methylation-God Kit (Cat\# D5006). The 392-bp fragment within the P16 exon-1 was amplified as previously described $(24,25)$. The 468 -bp fragment within the $P 16$ promoter was amplified with touchdown PCR $\left(65^{\circ} \mathrm{C}\right.$ to $\left.50^{\circ} \mathrm{C},-1^{\circ} \mathrm{C} / \mathrm{cycle}\right)$ using a $\mathrm{CpG}$-free primer set (forward, $5^{\prime}$-ggtgg ggttt ttata attag gaaag-3'; reverse, 5'-accct atccc tcaaa tcctc taaa-3'). The 392/468-bp PCR products were analyzed with DHPLC system (Transgenomics, USA) at the partial denaturing temperature of $58.6 / 55.5^{\circ} \mathrm{C}$, and confirmed with clone sequencing.

\section{Genome-Wide Analysis of DNA methylation}

Illumina Infinium HD Methylation 850K arrays were used to perform differential CpG methylation analyses on CCD-18Co cells stably transfected with the P16-Dnmt and PTRIPZ control vectors without doxycycline treatment according to the Assay Manual (by Capitalbio Technology Corporation, Beijing). DNA methylation ratio for probed $C p G$ sites were computed as the ratio of normalized methylated signal intensity to the sum of methylated and unmethylated signal intensities using GenomeStudio software. Using the control vector at passage- 5 as a reference, $\Delta$ methylation ratio was calculated to represent differential methylation for each CpG site in the baseline P16-Dnmt cells at passage-6, -9, and -13 . When the $\Delta$ methylation ratio for a $\mathrm{CpG}$ site in $\mathrm{CpG}$ islands around transcription start site (TSS-CGI) is more than $10 \%$ in the baseline P16-Dnmt cells at passage- 6 , and methylation ratio was consistently increased (at any level) in the baseline P16-Dnmt cells at passage-9 and -13 , the differential methylation was considered to be significant. When significant methylation was detected at 2 or more probed-CpG sites within a TSS-CGI, this CGI is defined as hypermethylated TSS-CGI. KEGG_PATHWAY enrichment was performed to analyze possible functions of differential methylation 
at probed $\mathrm{CpG}$ sites between cells with various treatments. The Methylation850K datasets were uploaded to NCBI with the accession number GSE111505.

\section{Western Blot and Confocal Analysis of P16 Expression Level}

P16 protein levels in cells were analyzed as previously described (8). Rabbit monoclonal antibody against human P16 protein (ab108349, Abcam, Britain) was used in Western blot assay and mouse monoclonal antibody against human P16 protein (Ventana Roche-E6H4, USA) was used in the immunostaining assay.

\section{Detection of the Length of Telomere by Quantitative PCR}

Copy number of the telomere gene was determined with quantitative real-time PCR (primer set: forward, 5'-cggtt tgttt gggtt tgggt ttggg tttgg gtttg ggtt-3'; reverse, 5'-ggctt gcctt accct taccc ttacc cttac cctta ccct-3') as described (26). Non-telomere gene 36B4 was used as reference (primer set: forward, 5'-cagca agtgg gaagg tgtaa tcc-3'; reverse, $5^{\prime}$-cccat tctat catca acggg tacaa-3'). The relative copy number was calculated using the formula $\left(2^{-\Delta C T}\right)$. Each experiment was performed in triplicate.

\section{Genome-Wide Analysis of Copy Number Variations}

The genome-wide copy number variation was analyzed through construction of Library using TruSeq Library Construction Kit and sequenced with Illumina HiSeq XTen by Novogene (Beijing). The average sequencing depth was about 10 (Supplementary Dataset file 1)

\section{$\beta$-Galactosidase Staining}

$\beta$-Galactosidase in situ Staining Kit (GENMED, Shanghai) was used according to the User Manual. The experiments were repeated at least two times. 
bioRxiv preprint doi: https://doi.org/10.1101/405407; this version posted August 30, 2018. The copyright holder for this preprint (which was not certified by peer review) is the author/funder, who has granted bioRxiv a license to display the preprint in perpetuity. It is made available under aCC-BY-NC-ND 4.0 International license.

\section{Statistical Analysis}

Results were displayed by constituent ratios of enumeration or ranked data. All $P$-values were

two-sided, and a difference with $P<0.05$ was considered statistically significant. 


\section{RESULTS AND DISCUSSION}

Recently, we constructed a P16-specific DNA methyltransferase P16-Dnmt with seven-zinc finger protein (7ZFP) that could specifically bind to and induce full methylation of whole $P 16 \mathrm{CpG}$ islands in cancer cells (8). To study the effect of $P 16$ methylation on cancer development, a model of full methylation of $P 16 \mathrm{CpG}$ islands in normal diploid cells first needed to be established. Therefore, normal human primary CCD-18Co fibroblasts were stably transfected with P16-Dnmt. In pilot study, P16-Dnmt expression was induced by addition of doxycycline into the medium (dox; final concentration, $0.25 \mu \mathrm{g} / \mathrm{mL}$ ). Three weeks after dox-treatment, a chromatographic peak for methylated-P16 alleles was observed in P16-Dnmt\&dox fibroblasts in DHPLC analysis (Figure 1A). Such methylation peak was not detected in baseline P16-Dnmt fibroblasts (without dox-treatment), 7ZFP\&dox, vector control\&dox, and parental mock cells. Bisulfite-sequencing confirmed these results (Figure 1B). The entire P16 CpG islands were extensively methylated in P16-Dnmt\&dox cells with an average methylation density of $51.4 \%$, and not methylated in vector control\&dox cells. Interestingly, a few methylated $\mathrm{CpG}$ sites (6.8\%) were also observed in baseline P16-Dnmt cells, indicating baseline P16-Dnmt expression in P16-Dnmt stably transfected fibroblasts without dox-treatment.

It is well known that the $P 16$ gene plays a crucial role in cell senescence (27-29). To investigate whether P16 methylation may prevent cell senescence, above pooled P16-Dnmt\&dox, vector control\&dox, and baseline P16-Dnmt control fibroblasts were continuously subcultured. Unexpectedly, both P16-Dnmt\&dox and vector control\&dox cells did not proliferate at passage-6 in the $10^{\text {th }}$ post-dox-treatment week, accompanied with cell size increase and cell disruption. As described blow, the same phenomenon was also observed in the baseline vector control cells without 
dox-treatment. In contrast, baseline P16-Dnmt fibroblasts remained to actively proliferate at this time point (Supplementary Figure 1). This suggests that baseline P16-Dnmt may be more efficient in preventing fibroblast senescence, probably due to less off-target effect or less cell stress in baseline P16-Dnmt cells.

To confirm whether baseline P16-Dnmt indeed prevents cell senescence and exclude the possible disturbance from dox-treatment, baseline P16-Dnmt fibroblasts and baseline vector control fibroblasts (without dox-treatment) were continuously observed using long-term IncuCyte live-cell imaging system with serial subcultures and medium renewals as described in the method section. The average proliferation rate of baseline P16-Dnmt cells (9 wells/group) was significantly higher than that of baseline vector control cells at the post-transfection subculturing number 5 (passage-5) (Figure 2, $p<0.001$ ). Notably, while baseline vector control fibroblasts stopped proliferating at passage-6, baseline $\mathrm{P} 16-\mathrm{Dnm}$ cells continued to proliferate until passage- 26 within the $53^{\text {th }}$ post-transfection week and did not become more abundant at later time points (Figure 3).

To evaluate the senescence status of these cells, $\beta$-galactosidase staining assay was used to detect senescing cells. The proportion of $\beta$-galactosidase-positive staining cells was significantly higher in baseline vector control cells than baseline P16-Dnmt cells at passage-5 (Figure 4, $p<0.001$ ). Together, these results indicate that baseline P16-Dnmt expression could promote proliferation of human primary fibroblasts and prevent cell senescence.

To study whether baseline P16-Dnmt indeed induce $P 16$ methylation in these fibroblasts, the methylation status of $P 16$ alleles in these cells were dynamically analyzed using Illumina Methylation850K array. The prevalence of methylated $\mathrm{CpG}$ sites were gradually increased at the 
probed $C p G$ sites within entire $P 16$ CpG islands in baseline P16-Dnmt CCD-18Co cells along with cell subculture (Figure 5A, red-dot-line squared). Such phenomenon was not observed in $P 14$ and $P 15$ CpG islands located within the same gene locus (Supplementary Figure 2A). Although hypermethylation was also found in most $\mathrm{CpG}$ sites within the MMP28 CpG islands, hypermethylation at only sporadic $\mathrm{CpG}$ sites were observed within non-target $\mathrm{CpG}$ islands, including CCNE2, $C D H 2$, GATA5, RUNX3, TERT, and WT1 genes (Supplementary Figure 2B). Because P16-Dnmt could methylate entire target $\mathrm{CpG}$ islands, thus, these sporadic CpGs may not be directly methylated by P16-Dnmt. Instead, hypermethylation at these sporadic CpGs may be one kind of adaptive response in fibroblasts escaping from senescence.

The DHPLC results further showed that the methylated- $P 16$ proportion was gradually increased in baseline P16-Dnmt cells along with cell subcultures ( 0 at passage- $3,20.2 \%$ at passage- 6 and $68.8 \%$ at passage-9; Figure 5B). Bisulfite-sequencing confirmed the Methylation850k and DHPLC analyses. The average methylation density was $28.1 \%$ and $41.6 \%$ at passage- 6 and passage- 9 , respectively, and further increased to $63.2 \%$ at passage-13 within the 468 -bp promoter CpG islands (Supplementary Figure 3A). Similar results were also observed within $P 16$ exon-1 region (Supplementary Figure 3B). The above results indicate that baseline P16-Dnmt could efficiently and specifically induce $P 16$ methylation in CCD-18Co cells.

The results of Western blot analysis in this study showed that $P 16$ expression was significantly lost in baseline P16-Dnmt cells at passage-6 (Figure 5C). In the confocal immuno-fluorescence analysis, no P16-positve cell was observed in baseline P16-Dnmt cells at passage- 6 and passage-9, while P16 protein was observed in the nucleus of parental mock and baseline vector control fibroblasts at 
passage-5 (Figure 5D). Generally $P 16$ expression is repressed in embryonic/tissue stem cells, induced-pluripotent stem cells (iPSC), and many immortalizing cells (30-33). Our results indicate that P16 expression is comprehensively inactivated in baseline P16-Dnmt cells, which prolongs the lifespan of fibroblast cells, reminiscent of the silencing of $P 16$ in the aforementioned cells.

To explore possible reason for the behavior differences between baseline P16-Dnmt and vector control cells, the length of telomeres was determined with quantitative PCR. The results unveiled that the average telomere length for baseline P16-Dnmt cells gradually decreased along with cell subculture $(66.4 \%$ and $24.5 \%$ at passage- 4 and passage -5 relative to vector control at passage- 4 ; Supplementary Figure 4). Interestingly, the KEGG_PATHWAY analysis results showed that top 3 pathways enriched with most differential methylation at Methylation850k dataset between vector control cells at passage- 5 and baseline P16-Dnmt cells at passage- 6 were metabolic pathways, pathways in cancer, and PI3K-Akt signal pathways (Figure 6A); between baseline P16-Dnmt cells at passage- 6 and passage-13, PI3K-Akt signal pathways, pathways in cancer, and metabolic pathways (Figure 6B).

We further investigate whether baseline P16-Dnmt or vector control cells can grow xenograft in NOD-SCID mice. As expected, no xenograft was formed in NOD-SCID mice subcutaneously injected both cells coated with Matrigel at the $17^{\text {th }}$ post-implantation week. A similar finding was also reported by others using TALE- or CRISPR/dCAS9-based tool to induce partial $P 16$ methylation $(18,21)$. The occurrence of gradually shorten telomere length in baseline P16-Dnmt cells and the inability to form xenograft in mouse may account for the idea that $P 16$ methylation could prevent the senescence and expand the lifespan, but could not immortalize human fibroblasts. However, 
oncogenic stimuli can induce oncogene-induced senescence in fibroblasts to protect against transformation, and $P 16$ inactivation/downregulation can prevent oncogene-induced cell senescence and thus sensitize cells to malignant transformation (34-37). It would be interesting to determine whether epigenetic $P 16$ inactivation may cooperate with other tumor-related genes or endogenous/environmental carcinogens to affect cancer development.

It was reported that $P 16$ could prevent centrosome dysfunction and genomic instability in primary cells and that shRNA-knockdown of $P 16$ expression generated supernumerary centrosomes through centriole pair splitting in human diploid epithelial cells (38-40). To study whether $P 16$ inactivation by DNA methylation affects genome stability of primary diploid fibroblasts, we analyzed the whole genome copy number changes with next-generation-sequencing. No detectable chromosome/gene copy number variation was detected between the genomes of baseline P16-Dnmt and vector control cells at passage-4 (Supplementary Dataset file 1).

Gene expression-controllable vectors such as pTRIPZ vector containing a "Tet-On" switch are often used in transfection studies to avoid uncontrollable gene overexpression. However, gene baseline expression in PTRIPZ vectors is unavoidable in cells even without dox-treatment (41-43). We found that only baseline P16-Dnmt cells could escape from senescing at the same time point at which P16-Dnmt\&dox and vector control cells stopped proliferating. That $68.8 \%$ P16 alleles were methylated in these cells indicates that the occurrence of baseline P16-Dnmt expression could lead to subsequent on-target DNA methylation. This also implies that gene baseline expression in pTRIPZ vectors might be a good strategy for gene function studies.

In conclusion, the present study unveils that $P 16$ methylation could prevent cell senescence, 
promote cell proliferation, and expand the life span of human fibroblasts, which may contribute to cancer development.

Conflict of Interest Statement: None declared.

\section{Abbreviations}

DHPLC, denatured high performance liquid chromatography; Dnmt, DNA methyltransferase;

P16-Dnmt, P16-specific ZFP-Dnmt; TSS, transcription start site; ZFP, zinc finger protein

\section{Acknowledgements}

This work was supported by Beijing Municipal Science and Technology (\#Z151100001615022) and the National Natural Science Foundation of China (\#81773036 and \#91640108) and the National Basic Research Program of China (973 \#2015CB553902). We appreciate Kendra Williams (USA) for English language editing.

\section{Author contribution}

YG performed the cell culture experiment. CC constructed the P16-Dnmt vectors. SX constructed vector for seven-zinc finger protein. BZ analyzed the methylation pattern. DD designed this study, analyzed the data, and wrote the manuscript. All authors have read and commented on the manuscript and approved the final version. 


\section{REFERENCES}

1. Serrano, M., et al. (1993) A new regulatory motif in cell-cycle control causing specific inhibition of cyclin D/CDK4. Nature, 366, 704-707.

2. Beroukhim, R., et al. (2010) The landscape of somatic copy-number alteration across human cancers. Nature, 463, 899-905.

3. Merlo, A., et al. (1995) 5' CpG island methylation is associated with transcriptional silencing of the tumour suppressor p16/CDKN2/MTS1 in human cancers. Nat Med, 1, 686-692.

4. Herman, J.G., et al. (1995) Inactivation of the CDKN2/P16/MTS1 gene is frequently associated with aberrant DNA methylation in all common human cancers. Cancer Research, 55, 4525-4530.

5. Deng, D., et al. (2010) Epigenetic Alterations as Cancer Diagnostic, Prognostic, and Predictive Biomarkers. In Herceg, Z., et al. (eds.), Epigenetics and Cancer, Pt B, vol. 71, pp. 125-176.

6. Kamb, A., et al. (1994) A cell cycle regulator potentially involved in genesis of many tumor types. Science, 264, 436-440.

7. Kamijo, T., et al. (1997) Tumor suppression at the mouse INK4a locus mediated by the alternative reading frame product p19ARF. Cell, 91, 649-659.

8. Cui, C., et al. (2015) P16-specific DNA methylation by engineered zinc finger methyltransferase inactivates gene transcription and promotes cancer metastasis. Genome Biology, 16, 252.

9. Gan Y., et al. (2018) Coordinated Transcription of ANRIL and P16 Genes Is Silenced by P16 DNA Methylation. Chinese Journal of Cancer Research 30, 93-103.

10. Sun, Y., et al. (2004) Methylation of $p 16$ CpG islands associated with malignant transformation of gastric dysplasia in a population-based study. Clinical Cancer Research, 10, 5087-5093.

11. Gao H., et al. (2017) Association between p16 methylation and malignant transformation of 
gastric dysplasia. Chinese Journal of Cancer Prevention and Treatment, 24, 431-436.

12. Jin, Z., et al. (2009) A multicenter, double-blinded validation study of methylation biomarkers for progression prediction in Barrett's esophagus. Cancer Res, 69, 4112-4115.

13. Hall, G.L., et al. (2008) p16 Promoter methylation is a potential predictor of malignant transformation in oral epithelial dysplasia. Cancer Epidemiol Biomarkers Prev, 17, 2174-2179.

14. Cao, J., et al. (2009) Methylation of p16 CpG Island Associated with Malignant Progression of Oral Epithelial Dysplasia: A Prospective Cohort Study. Clinical Cancer Research, 15, 5178-5183.

15. Liu, H.W., et al. (2015) P16 Methylation as an Early Predictor for Cancer Development From Oral Epithelial Dysplasia: A Double-blind Multicentre Prospective Study. EBioMedicine, 2, 432-437.

16. Zhang, B., et al. (2012) The p16-Specific Reactivation and Inhibition of Cell Migration Through Demethylation of CpG Islands by Engineered Transcription Factors. Human Gene Therapy,23, 1071-1081.

17. Z Zhang, B., et al. (2013) C-terminal in Sp1-like artificial zinc-finger proteins plays crucial roles in determining their DNA binding affinity. Bmc Biotechnology, 13, 106.

18. Bernstein, D.L., et al. (2015) TALE-mediated epigenetic suppression of CDKN2A increases replication in human fibroblasts. J Clin Invest, 125, 1998-2006.

19. Amabile, A., et al. (2016) Inheritable Silencing of Endogenous Genes by Hit-and-Run Targeted Epigenetic Editing. Cell, 167, 219-232.e14.

20. Stepper, P., et al. (2017) Efficient targeted DNA methylation with chimeric dCas9-Dnmt3a-Dnmt3L methyltransferase. Nucleic Acids Res, 45, 1703-1713.

21. Saunderson, E.A., et al. (2017) Hit-and-run epigenetic editing prevents senescence entry in primary breast cells from healthy donors. Nat Commun, 8, 1450. 
22. McDonald, J.l., et al. (2016) Reprogrammable CRISPR/Cas9-based system for inducing site-specific DNA methylation. Biol Open, 5, 866-74.

23. Qin, S.S., et al. (2014) Homeostatic Maintenance of Allele-Specific p16 Methylation in Cancer Cells Accompanied by Dynamic Focal Methylation and Hydroxymethylation. Plos One, 9, e97785.

24. Deng, D.J., et al. (2002) Simultaneous detection of CpG methylation and single nucleotide polymorphism by denaturing high performance liquid chromatography. Nucleic Acids Research, 30, E13

25. Luo, D.Y., et al. (2006) Methylation of CpG islands of $\mathrm{p} 16$ associated with progression of primary gastric carcinomas. Laboratory Investigation, 86, 591-598.

26. Gil, M.E., et al. (2004) Real-time quantitative PCR of telomere length. Mol Biotechnol, 27, $169-172$.

27. Alcorta, D.A., et al. (1996) Involvement of the cyclin-dependent kinase inhibitor p16 (INK4a) in replicative senescence of normal human fibroblasts. Proc Natl Acad Sci U S A, 93, 13742-13747.

28. Reznikoff, C.A., et al. (1996) Elevated p16 at senescence and loss of p16 at immortalization in human papillomavirus 16 E6, but not E7, transformed human uroepithelial cells. Cancer Res,56, 2886-2890.

29. Wang, W., et al. (2001) Characterization of regulatory elements on the promoter region of p16(INK4a) that contribute to overexpression of p16 in senescent fibroblasts. J Biol Chem,276, $48655-48661$.

30. Li, H., et al. (2009) The Ink4/Arf locus is a barrier for iPS cell reprogramming. Nature, 460, 1136-1139.

31. Hayashi, Y., et al. (2016) BMP-SMAD-ID promotes reprogramming to pluripotency by inhibiting 
p16/INK4A-dependent senescence. Proc Natl Acad Sci U S A, 113, 13057-13062.

32. Kan, C.Y., et al. (2012) Endothelial cell dysfunction and cytoskeletal changes associated with repression of p16(INK4a) during immortalization. Oncogene, 31, 4815-4827.

33. Farwell, D.G., et al. (2000) Genetic and epigenetic changes in human epithelial cells immortalized by telomerase. Am J Pathol, 156, 1537-1547.

34. Chang, Z., et al. (2014) Cooperativity of oncogenic K-ras and downregulated p16/INK4A in human pancreatic tumorigenesis. PLoS One, 9, e101452.

35. Kohsaka, S., et al. (2011) A population of BJ fibroblasts escaped from Ras-induced senescence susceptible to transformation. Biochem Biophys Res Commun, 410, 878-884.

36. Takeuchi, S., et al. (2010) Intrinsic cooperation between p16INK4a and p21Waf1/Cip1 in the onset of cellular senescence and tumor suppression in vivo. Cancer Res, 70, 9381-9390.

37. Wei, W., et al. (2003) Abolition of cyclin-dependent kinase inhibitor p16lnk4a and p21Cip1/Waf1 functions permits Ras-induced anchorage-independent growth in telomerase-immortalized human fibroblasts. Mol Cell Biol, 23, 2859-2870.

38. McDermott, K.M., et al. (2006) p16(INK4a) prevents centrosome dysfunction and genomic instability in primary cells. PLOS Biol, 4, e51.

39. Domínguez, D., et al. (2015) Centrosome aberrations in human mammary epithelial cells driven by cooperative interactions between p16INK4a deficiency and telomere-dependent genotoxic stress. Oncotarget, 6, 28238-28256.

40. Takayasu, T., et al. (2015) p16 Gene Transfer Induces Centrosome Amplification and Abnormal Nucleation Associated with Survivin Downregulation in Glioma Cells. Pathobiology, 82, 1-8.

41. Bohl, D., et al. (1997) Long-term control of erythropoietin secretion by doxycycline in mice 
bioRxiv preprint doi: https://doi.org/10.1101/405407; this version posted August 30, 2018. The copyright holder for this preprint (which was not certified by peer review) is the author/funder, who has granted bioRxiv a license to display the preprint in perpetuity. It is made available under aCC-BY-NC-ND 4.0 International license.

transplanted with engineered primary myoblasts. Nat Med, 3, 299-305.

42. Bieschke, E.T., et al. (1998) Doxycycline-induced transgene expression during Drosophila development and aging. Mol Gen Genet, 258, 571-579.

43. Chen, J., et al. (1998) Transgenic animals with inducible, targeted gene expression in brain. Mol

Pharmacol, 54, 495-503. 


\section{FIGURE LEGENDS}

Figure 1. Induction of P16 methylation in CCD-18Co fibroblasts by P16-Dnmt. (A) DHPLC chromatograms of methylated and unmethylated P16 exon-1 PCR products (392-bp) for fibroblasts stably transfected with P16-Dnmt or vector control, with or without doxycycline treatment for 3 weeks. Genomic DNA sample from P16-hemimethylated HCT116 cells was used as methylated- and unmethylated-P16 reference controls (P16M \& P16U Ctrls). (B) The results of bisulfite-sequencing of the 392-bp PCR products; each line represents a clone; red dots, methylated CpG sites; location of CpG sites within the amplicon was also displayed on the top. Methylation density was $51.4 \%$ (216/420) and 6.8\% (19/280) for P16-Dnmt\&dox and baseline P16-Dnmt cells, respectively.

Figure 2. Proliferation curves for baseline P16-Dnmt and baseline vector CCD-18Co cells at passage-5 in IncuCyte Zoom analysis. Phase object images of live cells for two groups taken on three different post-subculture/incubation days were also listed, respectively.

Figure 3. IncuCyte long-term observation of baseline P16-Dnmt and baseline vector control CCD-18Co cells. Both the post-transfection subculture day and passage number (subculture times) were labeled.

Figure 4. Comparison of the images and proportion of $\beta$-galactosidase-positive staining cells in baseline P16-Dnmt cells and baseline vector control cells at passage-5. Student $t$-test was used to calculate the $P$-value between the average ratio of $\beta$-galactosidase-positive staining cells to total CCD-18Co fibroblasts between baseline P16-Dnmt cells and baseline vector control cells in triplicate.

Figure 5. Effects of P16-Dnmt expression at baseline level on the methylation and expression status 
of $P 16$ alleles in CCD-18Co cells at different passage number. (A) Methylation status of each Illumina $850 \mathrm{~K}$ methylation array probed-CpG sites within various $P 16$ gene regions, including $\mathrm{N}$-shore (NS) and S-shore (SS), in baseline vector control cells (passage-5) and baseline P16-Dnmt cells (passage-6/9/13; *, CpG site with significant differential methylation). (B) DHPLC chromatograms of methylated and unmethylated $P 16$ promoter PCR products (468-bp) for fibroblasts stably transfected with P16-Dnmt or vector control. (C) Results of Western blot analysis for detecting the P16 protein (three lanes reorganized from the same original image). (D) Results of confocal immunostaining for detecting the P16 protein in situ.

Figure 6. Top 30 KEGG_PATHWAY pathways enriched with differential methylation at probed-CpG sites in Illumina $850 \mathrm{~K}$ methylation analysis between CCD-Co18 fibroblasts with and without baseline P16-Dnmt treatment. (A) between pTZ vector control cells at passage-5 and baseline P16-Dnmt cells at passage-6; (B) between baseline P16-Dnmt cells at passage- 6 and passage-13.

Supplementary Figure 1. Morphology of CCD-18Co fibroblasts stably transfected with P16-Dnmt or empty control vector, with or without doxycycline treatment at the $3^{\text {rd }}$ and $9^{\text {th }}$ post-doxycycline treatment week. Giemsa's in situ staining.

Supplementary Figure 2. Methylation ratios of probed $\mathrm{CpG}$ sites within baseline P16-Dnmt on-target and off-target CpG islands in baseline P16-Dnmt (at passage-6/9/13) and vector control CCD-18Co reference (at passage-5) in Illumina Methylation $850 \mathrm{~K}$ array analysis. The methylation status of CpG sites in CpG islands around transcription start site (TSS-CGI) and other regions within $P 16, P 14$ and P15 genes (A) and other TSS-CGls (B). NS, N-shore; SS, S-shore; NF, N-shelf; SF, S-shelf. Red mark (*), significantly hypermethylated probed-CpG site; Red line squared CpG sites, CpG sites within the 
TSS-CGI

Supplementary Figure 3. Bisulfite-sequencing for the 468-bp and 392-bp PCR products derived from the P16 Promoter (A) and Exon-1 (B) in CCD-18Co fibroblasts stably transfected with P16-Dnmt or empty control vector without doxycycline treatment at different passages. The listed average methylation density value was calculated according to ratio of actual methylated $\mathrm{CpG}$ sites to the total CpG methylation candidates within the informative clones in each group. The consensus sequences of these amplicons are illustrated on the top-lines. Each red dot represents a methylated CpG site. Each line represents a clone.

Supplementary Figure 4. Comparison of the average length of telomere in baseline P16-Dnmt and control CCD-18Co cells. Student $t$-test was used to calculate the $P$-value between the average telomere lengths for various CCD-18Co fibroblast groups (3 wells/group).

\section{Supplementary data}

Supplementary Dataset file 1. Genome-wide copy number analysis of CCD-18Co cells stably transfected with P16-Dnmt and control vector, with and without doxycycline treatment. 


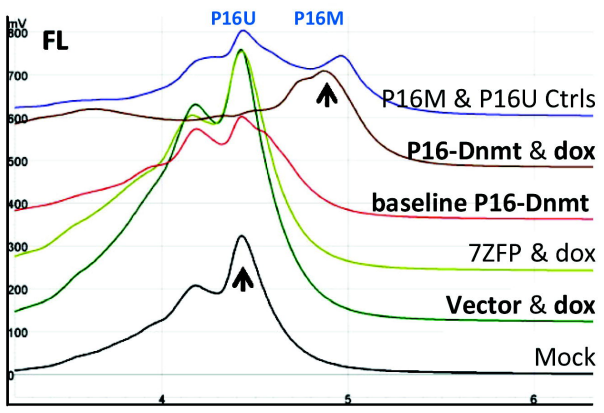

B

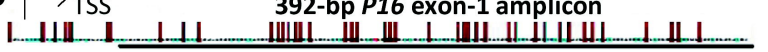
Retention time (min)

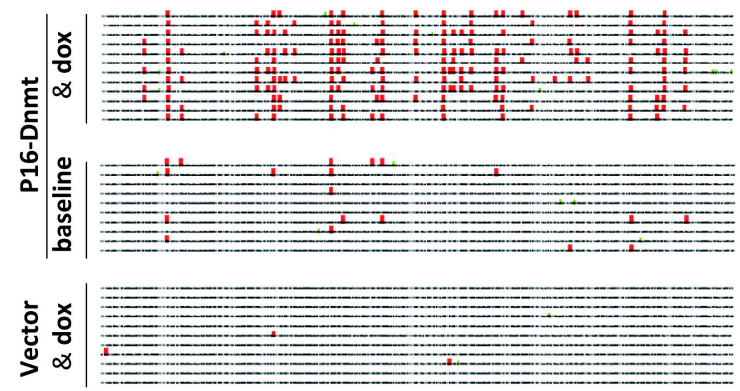

(Figure 1) 


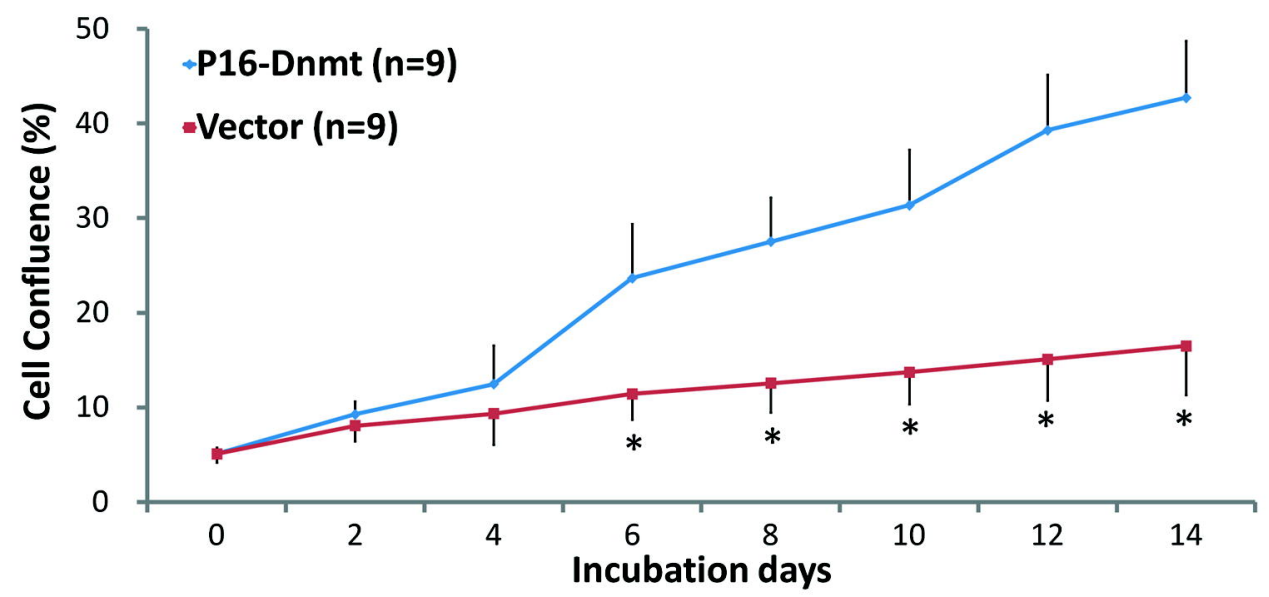

P16-Dnmt cells
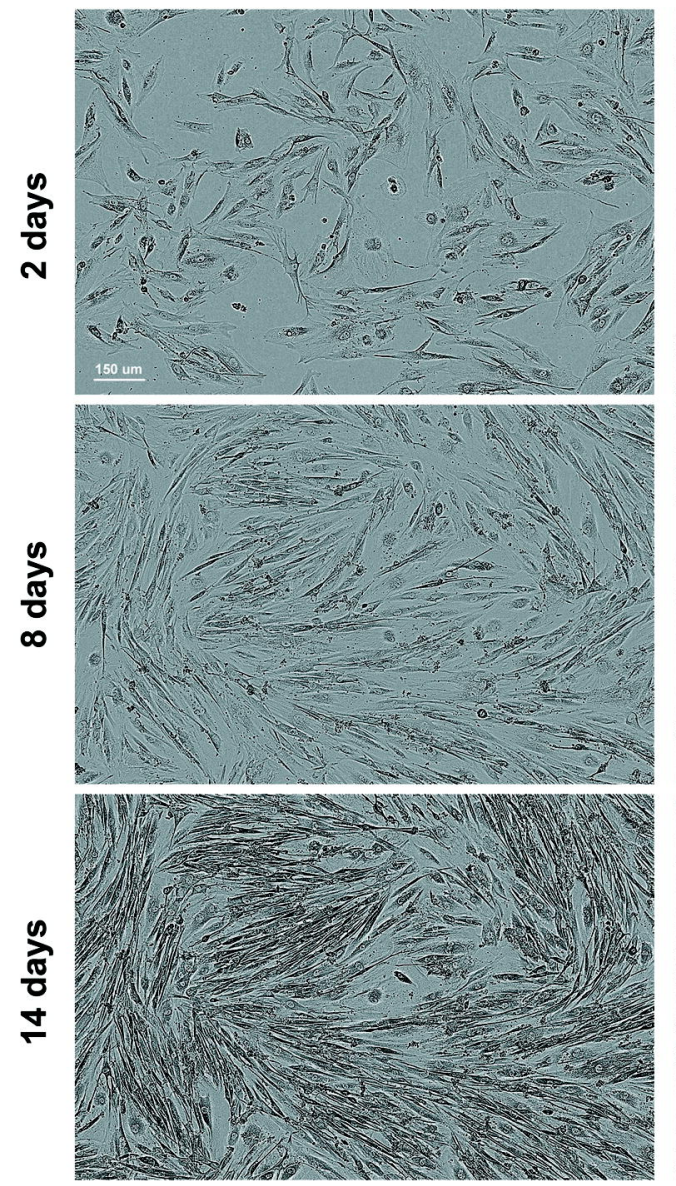

\section{Vector cells}
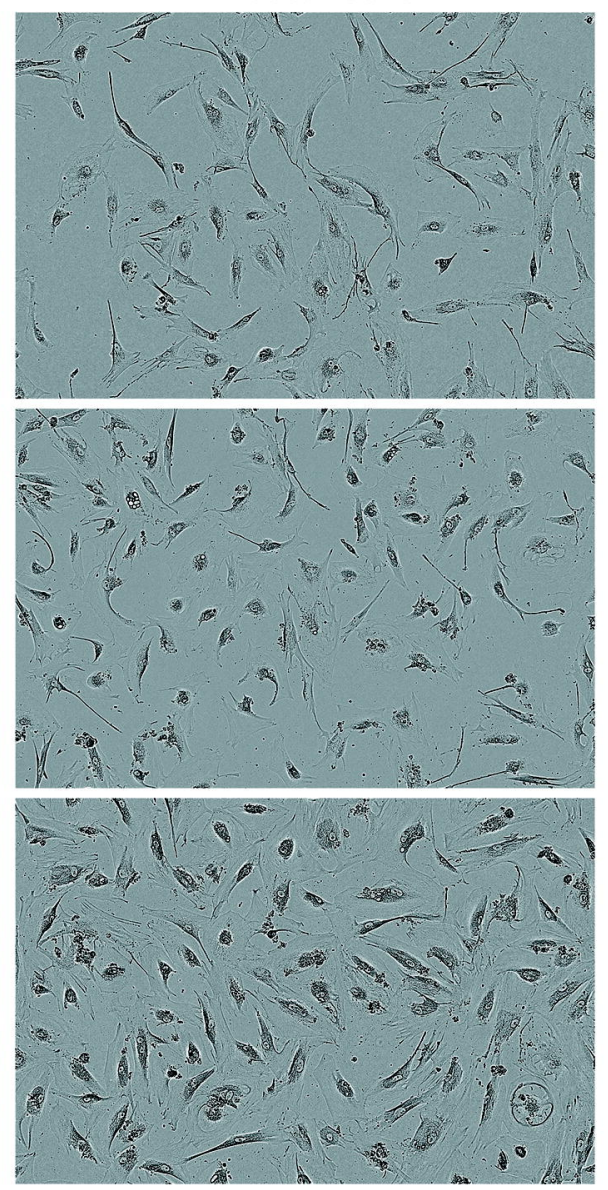

(Figure 2) 


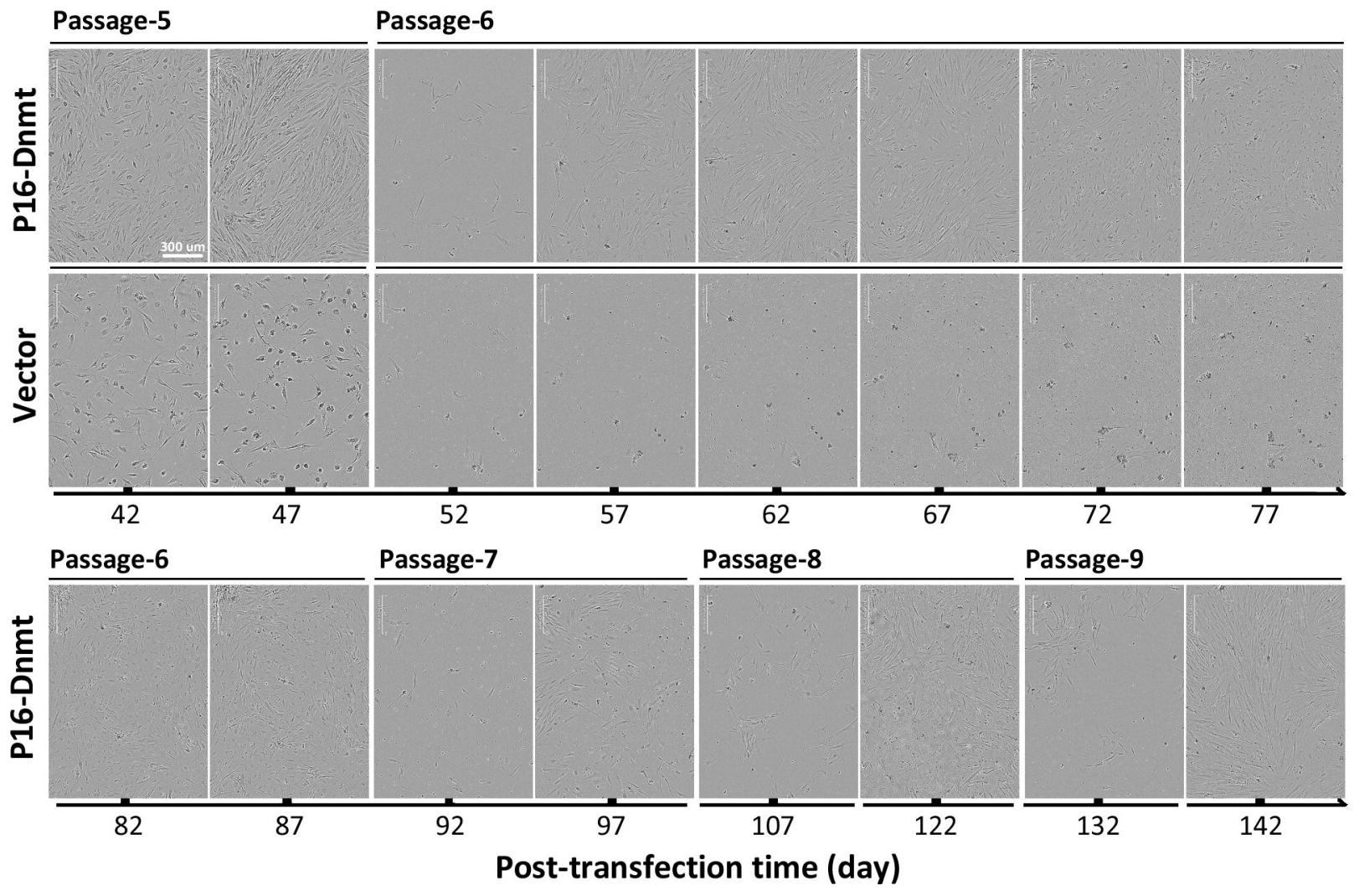

(Figure 3) 
Vector passage-5 a P16-Dnmt passage-6 a P16-Dnmt passage-9 $\square$ P16-Dnmt passage-13
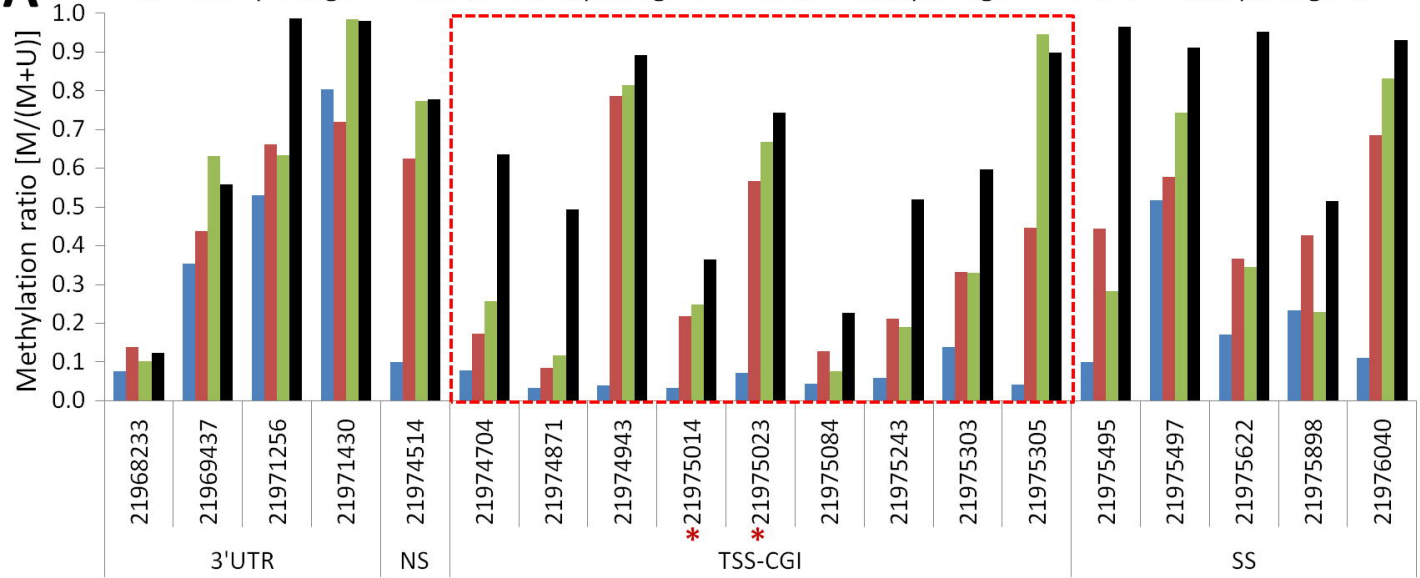

B

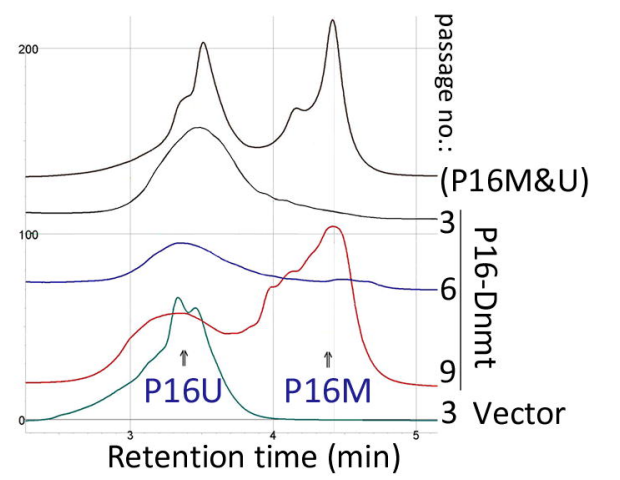

C

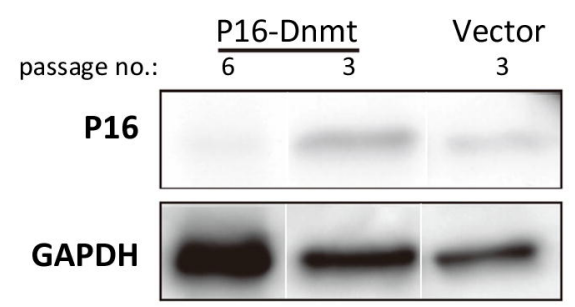

D

Mock

$\stackrel{0}{a}$

\section{P16-Dnmt}

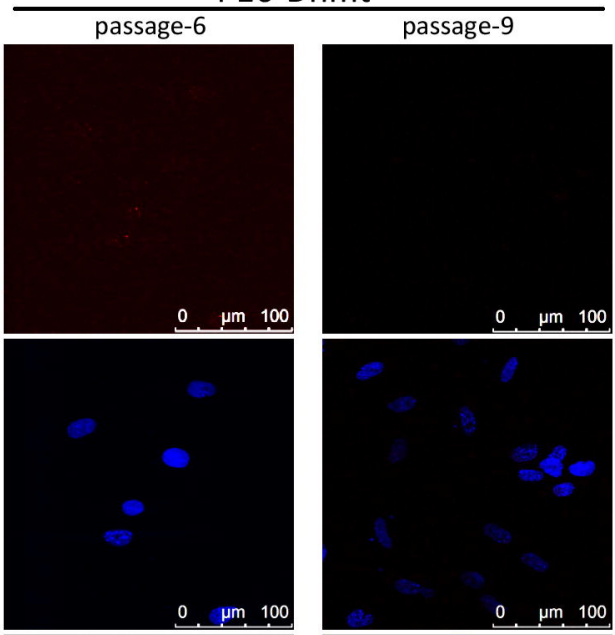

0 $\quad$ Im 100

0 In 100
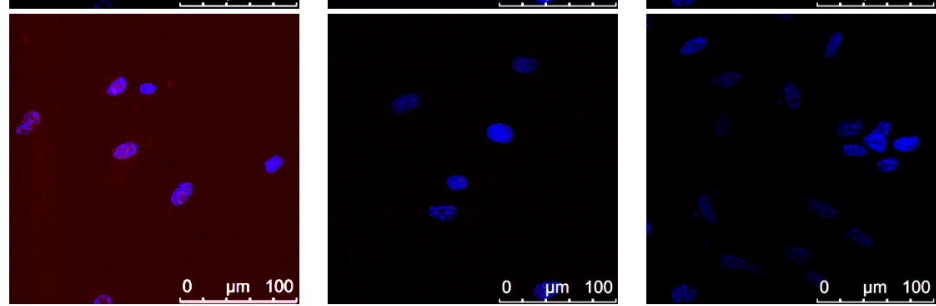

(Figure 5) 
Metabolic pathways | hsa01100 PI3K-Akt signaling pathway | hsa0415 Endocytosis | hsa0414 Rap1 signaling pathway | hsa04015
Focal adhesion | hsa04510

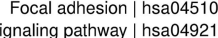
Oxytocin signaling pathway | hsa04921
Proteoglycans in cancer / hsa05205 Proteoglycans in cancer | hsa05205
Calcium signaling pathway | hsa04020 Ras signaling pathway | hsa04014 Neuroactive ligand-receptor interaction | hsa04080 HTLV-I infection | hsa05166 MAPK signaling pathway | hsa04010

@@Axon guidance | hsa04360 Inflammatory mediator regulation of TRP channels | hsa04750 Vascular smooth muscle contraction | hsa04270 Epstein-Barr virus infection | hsa05169 GMP-PKG signaling pathway | hsa04022 AMPK signaling pathway | hsa04152
Tight junction | hsa04530

Cell adhesion molecules (CAMs) / hsa04514 Glutamatergic synapse | hsa04724 Adrenergic signaling in cardiomyocytes | hsa0426
Phospholipase D signaling pathway | hsa04072

Sphingolipid signaling pathway | hsa04071

@@Wnt signaling pathway | hsa04310

Regulation of actin cytoskeleton | hsa04810

Hematopoietic cell lineage | hsa04640

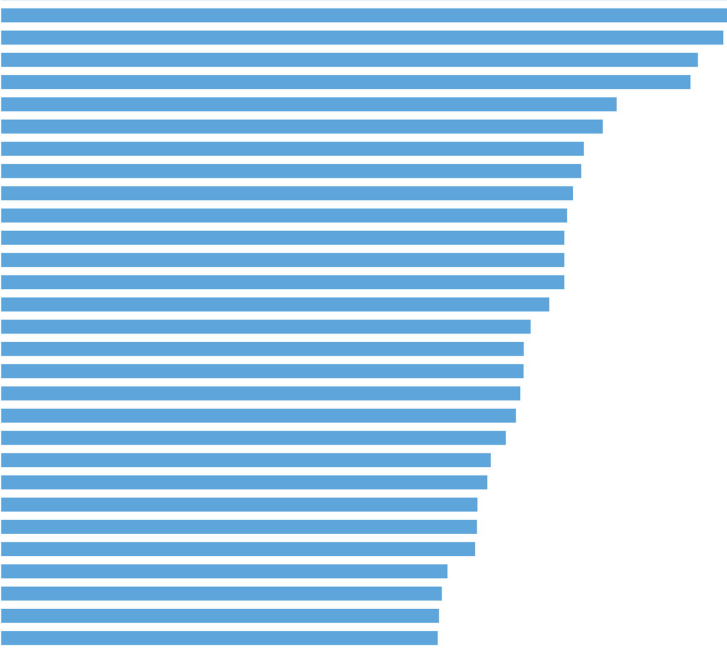

B (P16-Dnmt_passage-6 vs P16-Dnmt_passage-13)

Pl3K-Akt signaling pathway | hsa04151 $\frac{\text { Pathways in cancer } \mid \text { hsa05200 }}{\text { Metabolic pathways } \mid \text { hsa01100 }}$ Neuroactive ligand-receptor interaction | hsa04080 Rap1 signaling pathway | hsa04015 @@Axon guidance | hsa04360 MAPK signaling pathway | hsa04010 Cell adhesion molecules (CAMs) | hsa04514 Proteoglycans in cancer | hsa05205 cAMP signaling pathway | hsa04024 Oxytocin signaling pathway | hsa0492 Ras signaling pathway | hsa 0401 Phospholipase D signaling pathway | hsa04072 Platelet activation | hsa0461 Signaling pathways regulating pluripotency of stem cells | hsa0455 Vascular smooth muscle contraction | hsa04270 Adrenergic signaling in cardiomyocyles | hsa04261 @@Wnt signaling pathway | hsa04310 cGMP-PKG signaling pathway | hsa04022 AMPK signaling pathway | hsa04152 Hippo signaling pathway | hsa0439 Melanogenesis | hsa04916

ECM-receptor interaction | hsa04512

Glutamatergic synapse | hsa04724 Endocytosis | hsa04144
Amoebiasis | hsa0514

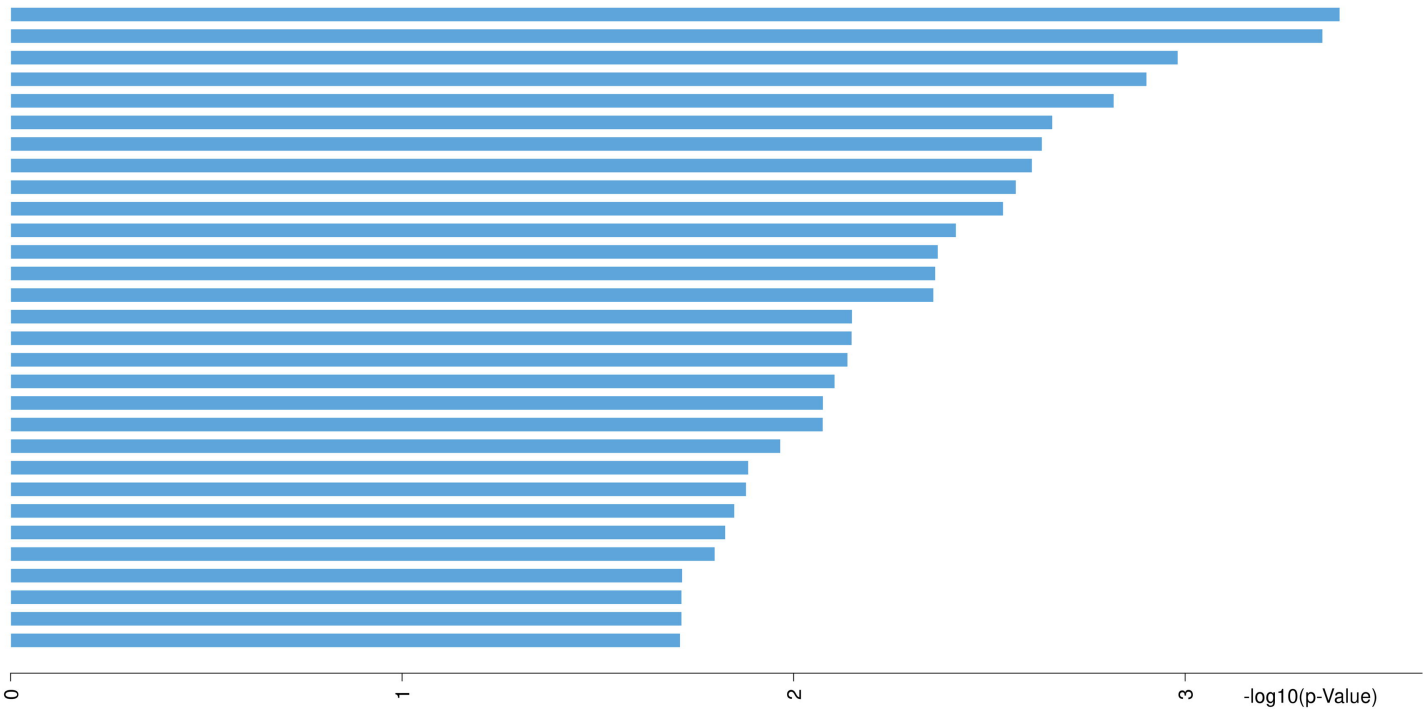

(Figure 6) 